\title{
Magnet Lattice Design for the Transmission of Power Using Particle Beams
}

\author{
Daniel Marley \\ Office of Science, Science Undergraduate Laboratory Internship (SULI) \\ North Carolina State University \\ SLAC National Accelerator Laboratory \\ Menlo Park, CA
}

12 August 2011

Prepared in partial fulfillment of the requirements of the Office of Science, Department of Energy's Science Undergraduate Laboratory Internship under the direction of James Welch at the SLAC Accelerator Directorate.

Participant:

Signature

Research Advisor:

Signature

Work supported by US Department of Energy contract DE-AC02-76SF00515. 


\section{TABLE OF CONTENTS}

Abstract

$\begin{array}{ll}\text { Introduction } & 1\end{array}$

$\begin{array}{lr}\text { Methods } & 3\end{array}$

$\begin{array}{lr}\text { Results } & 8\end{array}$

$\begin{array}{lr}\text { Discussion and Conclusions } & 9\end{array}$

$\begin{array}{lr}\text { Acknowledgments } & 10\end{array}$

$\begin{array}{ll}\text { References } & 11\end{array}$ 


\begin{abstract}
Magnet Lattice Design for the Transmission of Power Using Particle Beams. DANIEL MARLEY (North Carolina State University, Raleigh, NC 27695) JAMES WELCH (SLAC Accelerator Directorate, Menlo Park, CA 94025)

As the amount of electricity generated by renewable energy sources continues to increase, the current method of power transmission will not serve as an adequate method for transmitting power over very long distances. A new method for transmitting power is proposed using particle beams in a storage ring. Particle beams offer an incredibly energy efficient alternative to transmission lines in transmitting power over very long distances. A thorough investigation of the magnet lattice design for this storage ring is presented. The design demonstrates the ability to design a ring with stable orbits over a $381.733 \mathrm{~km}$ circumference. Double bend achromats and FODO cells are implemented to achieve appropriate $\beta$ functions and dispersion functions for 9-11 GeV electron beams.
\end{abstract}




\section{INTRODUCTION}

Although the manner for transmitting power in the United States is well established, there still exist some shortcomings of using transmission lines to efficiently transmit power over very long distances. This paper evaluates the current state of the electrical power system (the grid) and explores the idea of using particle beams for power transmission with a discussion of the magnet lattice that has been designed for such an endeavor.

\section{Background Information}

Electrical power is transmitted from power plants to consumers through various changes in voltage over multiple power lines. Transmission lines transfer most of this power over very long distances, at a few hundred kilovolts, from the power plants to substations. From the substations, lower-voltage (a few $\mathrm{kV}$ ) lines carry power to primary consumers and then pass through another step-down transformer (120 and 240V) before entering housing developments. The transmission line is a critical piece of the electrical grid in that it first receives the raw power from the generators and is responsible for distributing the power at a high efficiency to substations. The voltages at which these lines operate include 138, 230, 345, 500, and $765 \mathrm{kV}$. These transmission lines currently retain an average of about $93 \%$ efficiency and increasing the voltage is one way of improving the efficiency by minimizing resistive losses [1]. However, they still grow approximately linearly with distance and eventually become an unfavorable form of power transmission [2]. This property of transmission lines hinders the ability to share excess power with distant areas experiencing a power deficit. Transmission

lines are also becoming less capable of providing enough power to serve the increased demand from consumers [3]. 


\section{Motivation \& Purpose}

In recent years there has been an ongoing shift from fossil fuels to renewable energy sources. The two main types of renewable energy sources of interest are nuclear and solar power because they provide the most promise for widespread distribution. Nuclear power is already a popular energy source with 32 states in the continental United States (US) hosting at least one nuclear reactor [4]. While most of these reactors are located in the eastern half of the US, there is a surge of solar power production in the western half of the US, with California producing the most of any state [5]. Nuclear reactors, however, are being relocated to different areas of the country that contain a less dense population. As the efficiency and power production of these forms of renewable energy sources continue to grow, and investment in transmission lines continues to decline, there is an ever-increasing

need to transmit the power over very long distances. Solar power is generated largely in the desert of the southwestern US where the population density is very low. Because nuclear and solar power plants are being placed in locations were very few people live, there is a need to transmit the power they produce over very long distances. Transmitting this power over very long distances (from the power plants where no one lives to areas of high population density) motivates us to propose a new method for transporting power across the country, and possibly between continents. Fig. 1 depicts a schematic of this process and shows possible locations for generators and loads.

Particle accelerators are routinely used in high-energy physics experiments around the world. The particle beams that make these accelerators useful are tremendously energetic (up to $7 \mathrm{TeV}$ at the Large Hadron Collider) and typically run from a few $\mathrm{MeV}$ to a few $\mathrm{GeV}$, or five orders of magnitude greater than that of current transmission lines. Along with the tremendous voltages at which particle accelerators operate, they are also incredibly energy efficient. The primary source of energy loss is synchrotron radiation, which occurs when the 
particles undergo centripetal acceleration in a storage ring, described by (for electrons):

$$
P_{\text {lost }}=88.5 \frac{E^{4} I}{2 \pi \rho} \Delta \theta
$$

with the energy, $E$, measured in $\mathrm{GeV}$, the current, $I$, in amperes, and the radius of curvature, $\rho$, in meters. Incorporating particle beams into the grid will allow for cost-effective power distribution over incredibly long distances (e.g., around the United States or from North to South America). To adequately determine the feasibility and viability of using particle beams for power transmission, multiple factors need to be researched: the current condition of the grid, the energy efficiency of particle beams, the cost for materials and operation, the status of other proposed technologies compared to that of particle beams, and the design of the arrangements of magnets, or lattice, to focus and steer the beams. The work presented explores the magnet lattice design for the future electron storage ring for particle transmission.

\section{METHODS}

To design the magnet lattice for an electron storage ring, the transfer matrix method was applied to generate ring designs and analyze the electron stability. The following section describes the mathematical formalism of the transfer matrix method applied to design the magnet lattice and the software used to calculate beam stability and the lattice design. The lattice design was created for an example ring that nearly encloses the United States,

joining the Eastern, Western, and Texas interconnects. The size of this ring represents the magnitude and scope of this project to efficiently transmit power over very long distances. 


\section{Transfer Matrix Method}

In an electron storage ring, electrons remain in orbit due to magnetic forces from dipole, quadrupole and even sextupole magnets. These magnets steer and focus the electrons around the ring with an approximately Gaussian distribution about the ideal orbit. To quantify the behavior and properties of the electrons, a unique coordinate system has been defined with respect to the ideal orbit: $\hat{s}$ along the beam line, $\hat{x}$ in the radial direction, and $\hat{y}$ in the transverse direction, as noted in (Fig. 2) [6].

The lattice, or series of magnets and drift spaces (areas free of magnetic forces) that define an accelerator, design in this ring currently contains only quadrupoles and dipoles, although sextupoles can be added later if deemed necessary. The effect that these magnets have on electrons can be calculated using $K$, the focusing function:

$$
K_{x} \equiv \frac{1}{\rho^{2}}-\frac{e c}{E} B_{1}(s) \quad \& \quad K_{y} \equiv \frac{e c}{E} B_{1}(s)
$$

with $B_{1}(s)=\partial B_{y} / \partial x$, e the electron charge, $c$ the speed of light, and $E$ the energy of the particle of interest [7]. The focal length for the quadrupole magnets is $f=1 /(K l)$, with $l$ the length of the quadrupole. The focus/defocus character of quadrupole is a result of $\nabla \times \mathbf{B}=0$ so $d B_{x} / d y=-d B_{y} / d x$. That is a positive gradient in one direction implies a negative gradient in the other. In the limit of small oscillations, the linear equations of motion for an electron in an accelerator can be formed and then solved to reveal the position and phase functions based on the focusing function and structure of the lattice. The linear equation of motion is $x^{\prime \prime}+K_{x}(s) x=0$ (can substitute $x$ with $y$ and the derivatives are taken 
with respect to $s$ ) and the corresponding position solutions are [7]

$$
x(s)= \begin{cases}a \cos (\sqrt{K} s+b) & K>0 \\ a s+b & K=0 \\ a \cosh (\sqrt{|K|} s+b) & K<0\end{cases}
$$

The solutions produced will combine into a pseudo-periodic function that does not repeat itself exactly, but does follow an approximate sine/cosine shape.

For a given set of initial conditions, $x\left(s_{0}\right)$ and $x^{\prime}\left(s_{0}\right)$, a matrix equation can be written that describes the current position, $x(s)$, and phase, $x^{\prime}(s)$, functions due to the beam elements (quadrupole, dipole, or drift space)

$$
\mathbf{x}(s) \equiv\left(\begin{array}{c}
x(s) \\
x^{\prime}(s)
\end{array}\right) \quad \mathbf{x}(s)=\overleftrightarrow{\mathbf{M}}\left(s, s_{0}\right) \mathbf{x}\left(s_{0}\right)
$$

The form of this equation provides the necessary information for determining the transfer matrix, $\overleftrightarrow{\mathrm{M}}$ for each beam element.

$$
\begin{array}{ll}
K>0: & \overleftrightarrow{\mathbf{M}}\left(s, s_{0}\right)=\left(\begin{array}{cc}
\cos (\sqrt{K} l) & \frac{1}{\sqrt{K}} \sin (\sqrt{K} l) \\
-\sqrt{K} \sin (\sqrt{K} l) & \cos (\sqrt{K} l)
\end{array}\right) \\
K=0: & \overleftrightarrow{\mathrm{M}}\left(s, s_{0}\right)=\left(\begin{array}{ll}
1 & l \\
0 & 1
\end{array}\right) \\
K<0: & \overleftrightarrow{\mathrm{M}}\left(s, s_{0}\right)=\left(\begin{array}{cc}
\cosh (\sqrt{|K|} l) & \frac{1}{\sqrt{|K|}} \sinh (\sqrt{|K|} l) \\
\sqrt{|K|} \sinh (\sqrt{|K|} l) & \cosh (\sqrt{|K|} l)
\end{array}\right)
\end{array}
$$


For the sector dipole magnet of length $l, K>0\left(=1 / \rho^{2}\right)$ and the matrix simplifies to

$$
\overleftrightarrow{\mathrm{M}}\left(s, s_{0}\right)=\left(\begin{array}{cc}
\cos (l / \rho) & \rho \sin (l / \rho) \\
-\frac{1}{\rho} \sin (l / \rho) & \cos (l / \rho)
\end{array}\right)
$$

For $n$ beam elements, Eq. (4) can be rewritten as

$$
\mathbf{x}(s)=\left[\overleftrightarrow{\mathrm{M}}_{1}\left(s_{1}, s_{0}\right) \cdot \overleftrightarrow{\mathrm{M}}_{2}\left(s_{2}, s_{1}\right) \cdot \overleftrightarrow{\mathbf{M}}_{3}\left(s_{3}, s_{2}\right) \cdots \overleftrightarrow{\mathrm{M}}_{n}\left(s, s_{n-1}\right)\right] \mathbf{x}\left(s_{0}\right)
$$

This matrix product results in a unit matrix [7]. Not only does this help calculate final position and phase functions, but also the beam stability is contained within this matrix. If the Trace of the final matrix is less than or equal to $2(|\operatorname{Trace}(\overleftrightarrow{\mathrm{M}})| \leq 2)$ there exists a stable orbit. This result is derived from the requirement that the eigenvalues of the matrix satisfy the equations: $\lambda_{1}=1 / \lambda_{2}, \lambda_{1}+\lambda_{2}=\operatorname{Trace}(\overleftrightarrow{\mathbf{M}})$, and $\lambda^{2}-\operatorname{Trace}(\overleftrightarrow{\mathrm{M}}) \lambda+1=0$ for either eigenvalue [9]. This has to be calculated for both $x$ and $y$ to determine stability in both directions.

\section{Simulation Process \& Beam Parameters}

With this theoretical formalism in place, Mathematica 8 was used to compute the final matrix produced from the beam elements. Because the trace of a matrix is easily determined, the stability of the beams can be calculated in a quick and efficient manner. This allows for the calculation and frequent manipulation of beam parameters to fully optimize the design and explore many options in very little time. To calculate the final matrix, the parameters of the beam need to be defined. These parameters are collected in (Table 1).

After determining the beam stability in Mathematica, the Methodical Accelerator Design (MAD) software (v 8.52), developed by CERN, was used to input the lattice design and determine the $\beta$ functions in $x$ and $y$, along with the dispersion relation and an output of the 
coordinates of each beam element. The $\beta$ functions are very important in accelerator physics and are derived from the general solution to the linear equation of motion $x^{\prime \prime}+K_{x}(s) x=0$ :

$$
x(s)=\sqrt{\varepsilon \beta} \cos (\phi-\theta)
$$

where $\varepsilon$ is the emittance of the beam (area in phase space) and $\beta$ describe the lateral focusing properties of the lattice. From this equation one can see the maximum amplitude of the beam is $\sqrt{\varepsilon \beta}$ ( $\equiv \sigma$, the standard deviation). For this lattice, the amplitude should remain below $1 \mathrm{~mm}$ to ensure the beam does not become too wide and begin to be lost to the enclosure and other nonlinear effects. MAD can determine more complex features of the beam (nonlinear terms, beam position monitors, horizontal and vertical kickers, RF cavities, etc.) than Mathematica, and allows the user to input constraints on the system. MAD is also capable of determining stability, but the location of the instability is unknown and difficult to find. Being able to input constraints allows the user to have some freedom in writing a lattice because MAD can calculate the optimal values. However, in some instances the determined values are not ideal, and it is best to re-compute them using Mathematica.

It was pre-determined that there would be two main lattice elements for the beam: Double bend achromat (DBA) and a FODO cell. The best mechanism for controlling the dispersion of the beam was to implement a DBA. Two dipole magnets with a focusing quadrupole magnet, in $\hat{x}$, placed between them comprise a DBA. The usefulness of a DBA is that it prevents electrons of different energies that travel through the dipole magnet from taking a different path by eliminating dispersion outside of the DBA. As electrons come through the first dipole, they are refocused by the quadrupole to pass through the second dipole and emerge back in the electron bunch as if there had been no bend at all (Fig. 3). Outside of a DBA it is optimal to include either a doublet or a triplet of quadrupoles to ensure the beam is not lost and that it remains stable while entering and exiting a DBA [9]. Multiple 
drift cells are needed to cover the large distances planned by this ring. A cell that consists of only drift sections and quadrupoles is known as a FODO cell (a segment of a storage ring that is symmetric about its center), outlined in Fig. 4. These two design elements were implemented into Mathematica and MAD to yield a stable orbit of electrons around a storage ring.

\section{RESULTS}

After applying the transfer-matrix formalism in Mathematica and then inputing the results into MAD, three separate cell designs were combined to create a single lattice design. Two stable cells were created that incorporated a smaller bend radius (三 Cell 1) and a larger bend radius ( $\equiv$ Cell 2) through two different DBAs, and a third FODO cell (三 Cell 3) was created. The plots of the $\beta$ functions can be seen in Fig. 5,6 \& 7 .

Fig. 5 (Cell 1) represents the DBA with a smaller radius, Fig. 6 (Cell 2) the DBA with a bigger radius, and Fig. 7 (Cell 3) an example drift section. Table 2 displays the maximum $\beta$ functions in $x$ and $y$, the total length, and maximum dispersion for each Cell. The $\beta$ functions, dispersion, and total length vary between the different cells because they have different quadrupoles, drift spaces, and dipoles. Both Cell 1 and 2 have multiple quadrupoles implemented into the design. They have different dipoles form one another to achieve different bend angles as well. Because Cell 3 is just a FODO cell, the quadrupoles all consist of the same strength and the same separation between them. From the values used to create these cells, it is possible to see the wide variety in values necessary to gain stability in the electron orbit simply based on the type of DBA needed.

After the files were created and the stability was verified in Mathematica, MAD was used to combine these cells into one final ring design (Fig. 8). The ability to vary quantities and set constraints in MAD made it possible to match $\beta$ functions across the various cells. 
Constraints were also applied to ensure the dispersion remained zero outside of the DBAs and to attempt to keep the $\beta$ functions below $2000 \mathrm{~m}$. A stable beam was created that is $2355.38 \mathrm{~m}$ long, a maximum dispersion of $0.099596 \mathrm{~m}$ and a maximum $\beta_{x}$ and $\beta_{y}$ of 2188.89 $\mathrm{m}$ and $2254.96 \mathrm{~m}$, respectively. These values are not below the desired maximum, but exceed that value by less than $15 \%$ each.

\section{DISCUSSION AND CONCLUSIONS}

From the results it was possible to find an electron storage ring that would yield stable electron motion and cover approximately $381.733 \mathrm{~km}$. This length is short of the desired $10,000 \mathrm{~km}$, but with further work on the MAD program 10,000 km should be easily achievable by implementing more FODO cells and multiple DBAs. The power of MAD to iterate through the beam elements and vary them such that the constraints could be met allowed for the design to be created.

The desired parameters for this lattice made it particularly difficult in finding a large enough ring that produced a stable orbit. As the lattice became longer and longer, the $\beta$ functions became larger and larger. The size of the FODO cells were restricted by the size of the $\beta$ functions. The DBAs also became affected by the $\beta$ function size as well. Meeting the dispersion requirement was completed by varying the strength of the quadrupole in the DBA; the $\beta$ function would grow too large if the quadrupole was too far or too close to the surrounding dipoles. For future designs, allowing the $\beta$ functions to increase may make it possible to more quickly find a stable orbit for the ring. Also, as a result of the given parameters, the electron beam will contain greater than $300 \mathrm{MJ}$ worth of energy. This is a tremendous amount of energy traversing the beam and how this energy should be dissipated needs to be addressed in future beam designs. Regardless of the difficulties in meeting the pre-determined parameters, MAD made it much easier than expected to find a stable orbit 
because of its ability to vary parameters and constrain variables.

This lattice design is not the only possible design. If more constraints are revealed, or if some can be removed, the values that were formed for the quadrupoles, dipoles, and drift sections may be changed to yield different lattice designs. Some of these possible constraints may include the strength of permanent magnets that can be manufactured, the width of the pipe and tunnel, and the cost of tunneling, among others. From here there are multiple steps that should be taken to turn this example lattice into the final design including nonlinear terms, RF cavities, vertical bends to match the curvature of the earth, and creating more complex DBA cells. It is also important that the terrain be more thoroughly analyzed and a ring be designed that can more efficiently navigate the landscape.

With this design, a first step in demonstrating the possibility of this project has been taken and it proves the feasibility in at least achieving stability in a ring of this magnitude. Transmitting power over very large distances has gained plausibility from this design and encourages the exploration of other factors in developing this technology, such as the cost of construction, manufacturing, cost of operation, and economic advantage over current transmission lines and other competing technologies.

\section{ACKNOWLEDGMENTS}

This work was supported by the U.S. Department of Energy Office of Science through the Summer Undergraduate Laboratory Internship (SULI) Program. I am very appreciative of my mentor Jim Welch for not only the opportunity to work on this interesting and very challenging project, but for also helping me continuously throughout the summer and better exposing me to accelerator physics. I would like to thank the director, Steve Rock, for providing a positive learning environment, and for exposing me to many new areas of physics. 


\section{REFERENCES}

[1] U.S. Energy Information Administration. (2009, November 19). Data on electricity transmission and distribution losses. Retrieved from http://www.eia.gov/tools/faqs/faq.cfm?id=105\&t=3

[2] L. Paris, G. Zini, M. Valtorta, G. Manzoni, and N. De Franco (1984). Proceedings from International Conference on Large High Voltage Electric Systems, 1984 Session: Present Limits of Very Long Distance Transmission Systems. Paris, France: Global Energy Network Institute.

[3] Schewe, P.F. (2007). The Grid: A Journey Through the Heart of Our Electrical World. Washington, DC: Joseph Henry Press

[4] U.S. Nuclear Regulatory Commission. (2011, May 19). Operating Nuclear Power Reactors. Retrieved from http://www.nrc.gov/info-finder/reactor/

[5] Gelman, R., Hummon, M., McLaren, J., and Doris, E. (2010, October). 2009 US State Clean Energy Data Book. U.S. Department of Energy: Washington, DC.

[6] Sands, M. (1970). The Physics of Electron Storage Rings: an introduction. Prepared for the US Atomic Energy Commission under contract no. AT(04-3)-515.

[7] Lee, S.Y. (1999). Accelerator Physics. River Edge, NJ: World Scientific Publishing Co. 


\section{FIGURES}

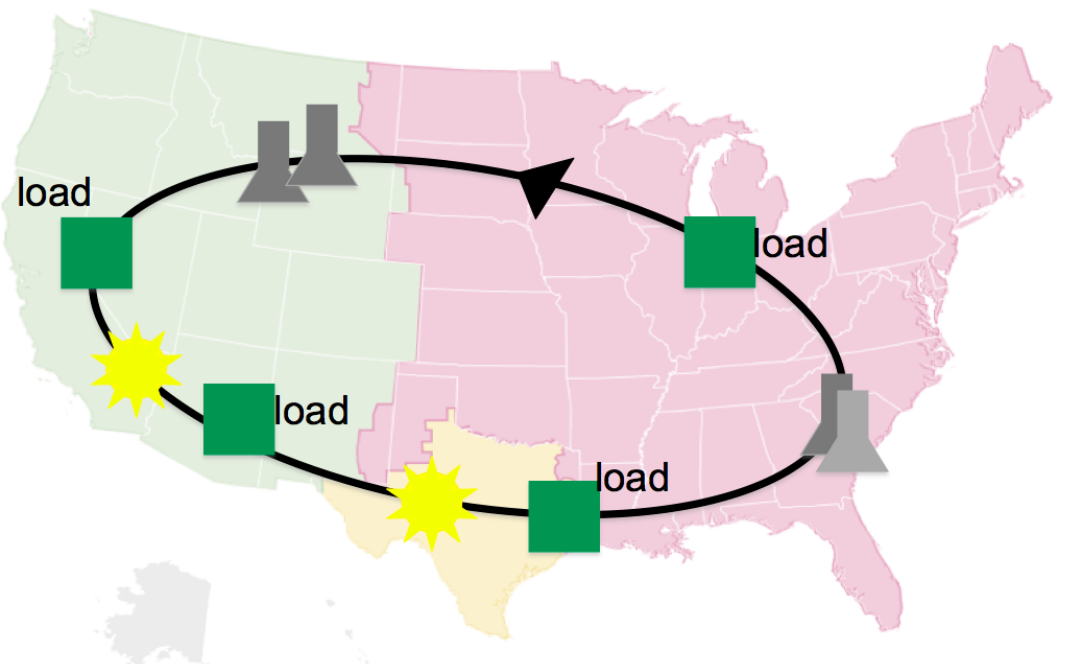

Figure 1: Outline of final storage ring with possible generator and load locations. Overlaid on a map of the US with the 3 interconnects colored: East (Pink), West (Green), Texas and (Orange).

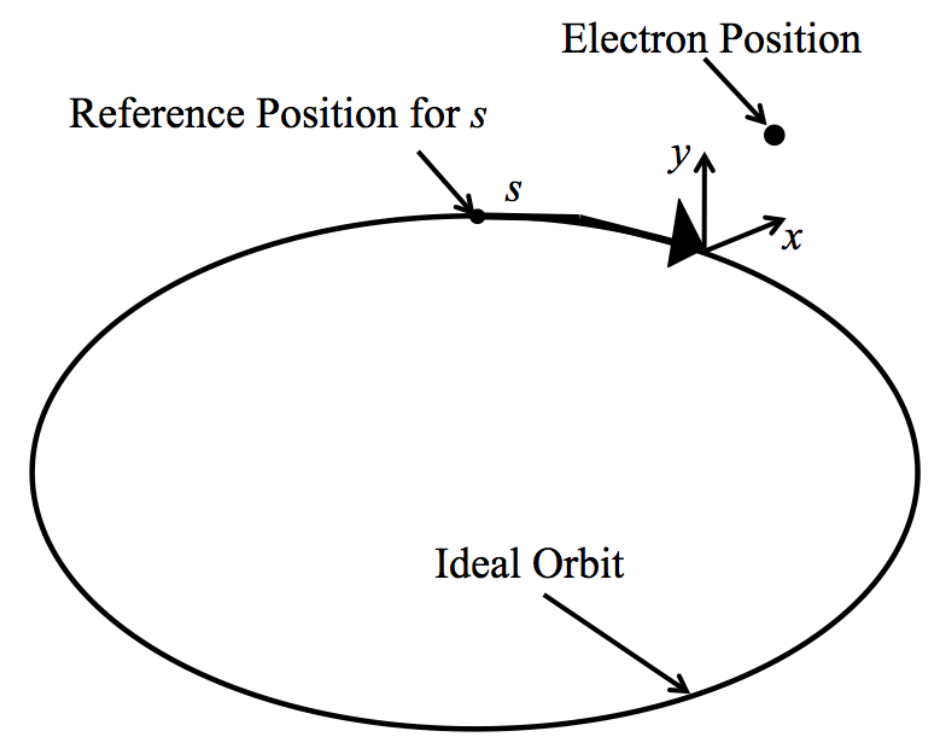

Figure 2: Schematic depicting the coordinate system for electrons in a storage ring. 


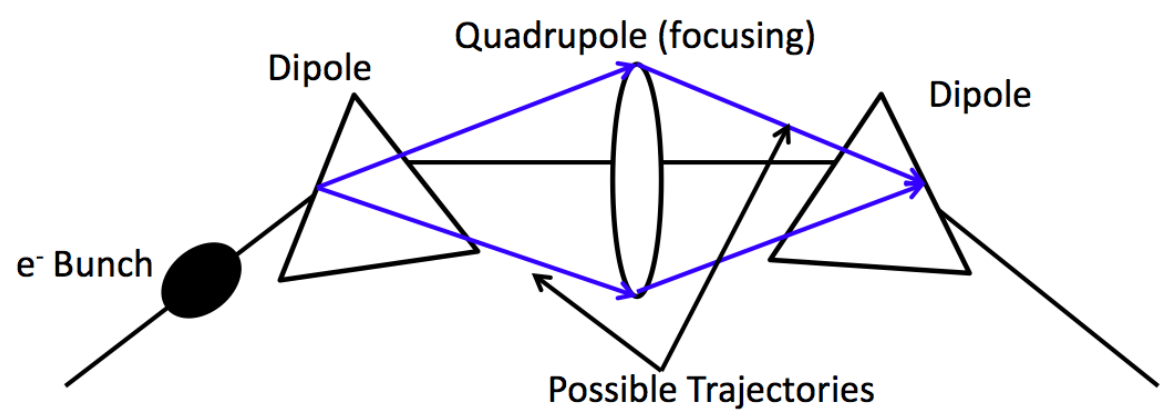

Figure 3: Schematic of a double bend achromat (DBA).

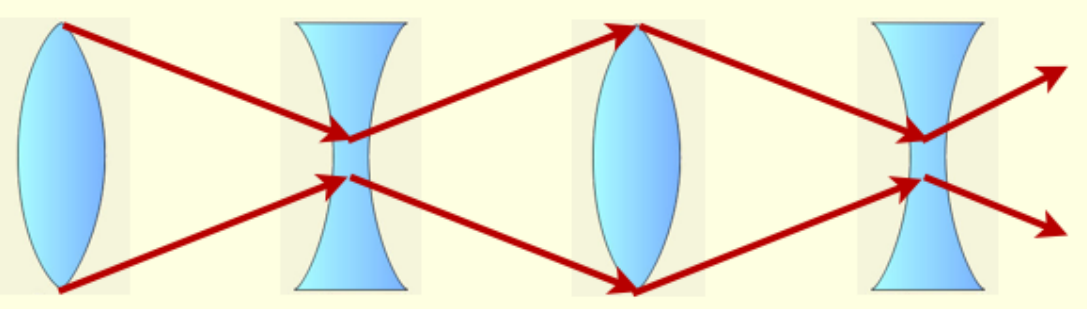

Figure 4: Diagram of a FODO lattice using optical lenses to represent quadrupoles. The spaces between each lens represent the drift spaces in a lattice. 


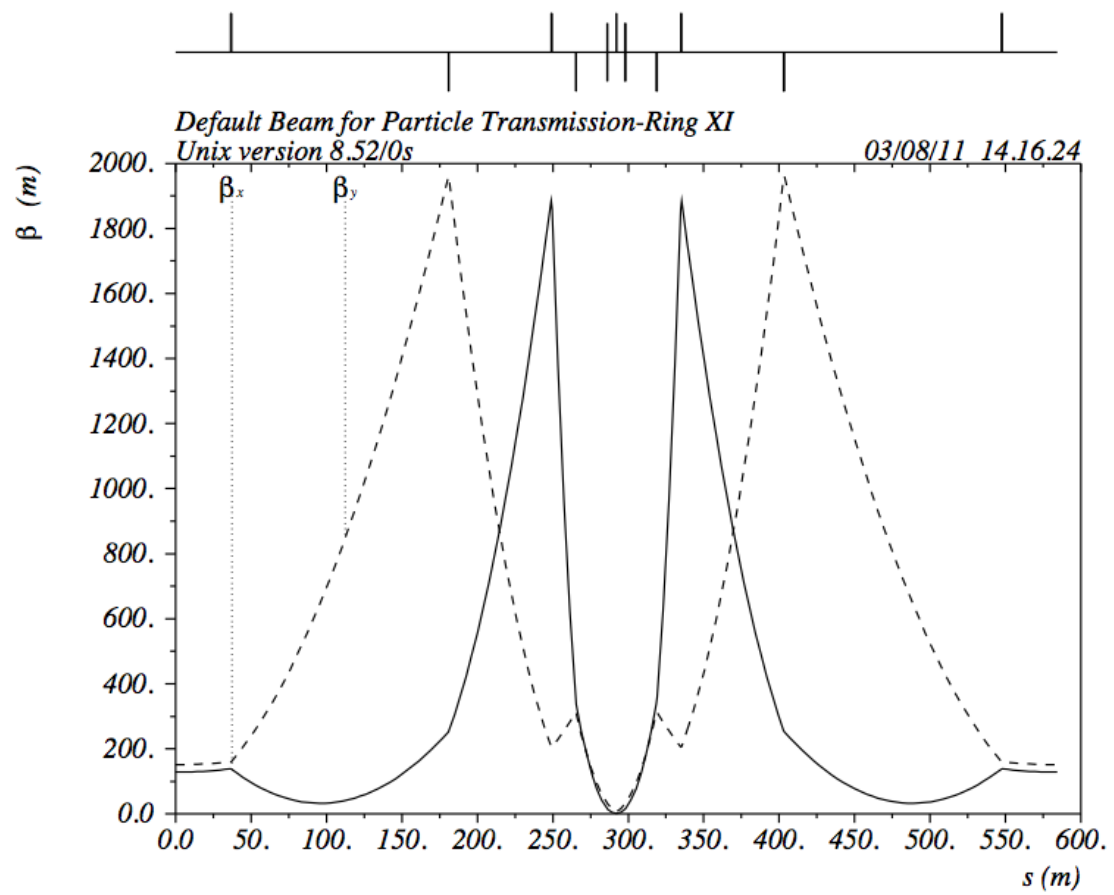

Figure 5: $\beta$ function for stronger DBA, smaller radius.

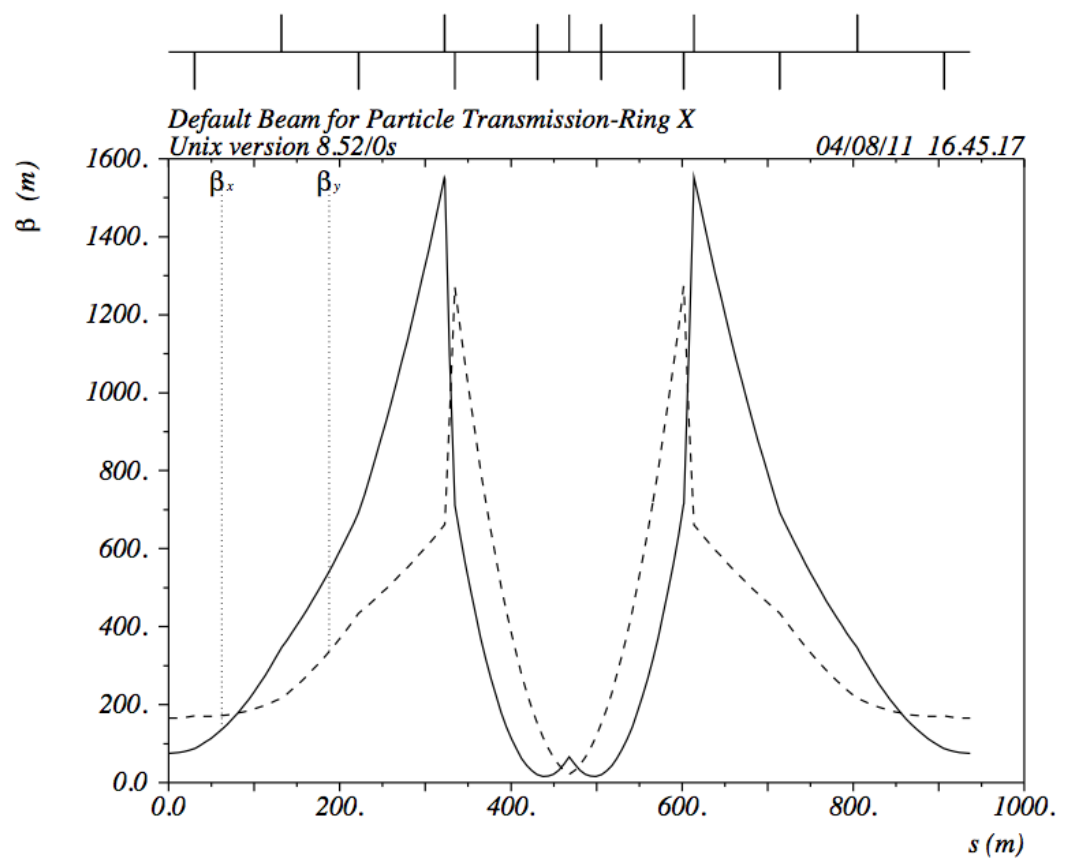

Figure 6: $\beta$ function for weaker DBA, larger radius. 


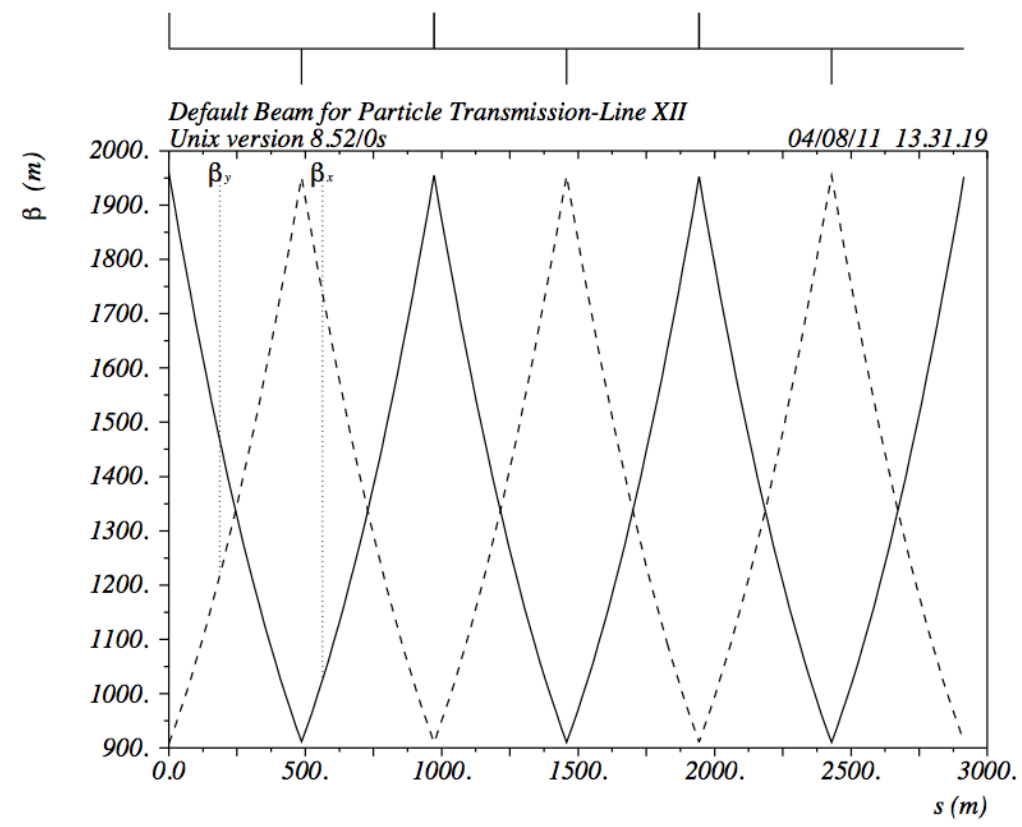

Figure 7: $\beta$ function for FODO cell.

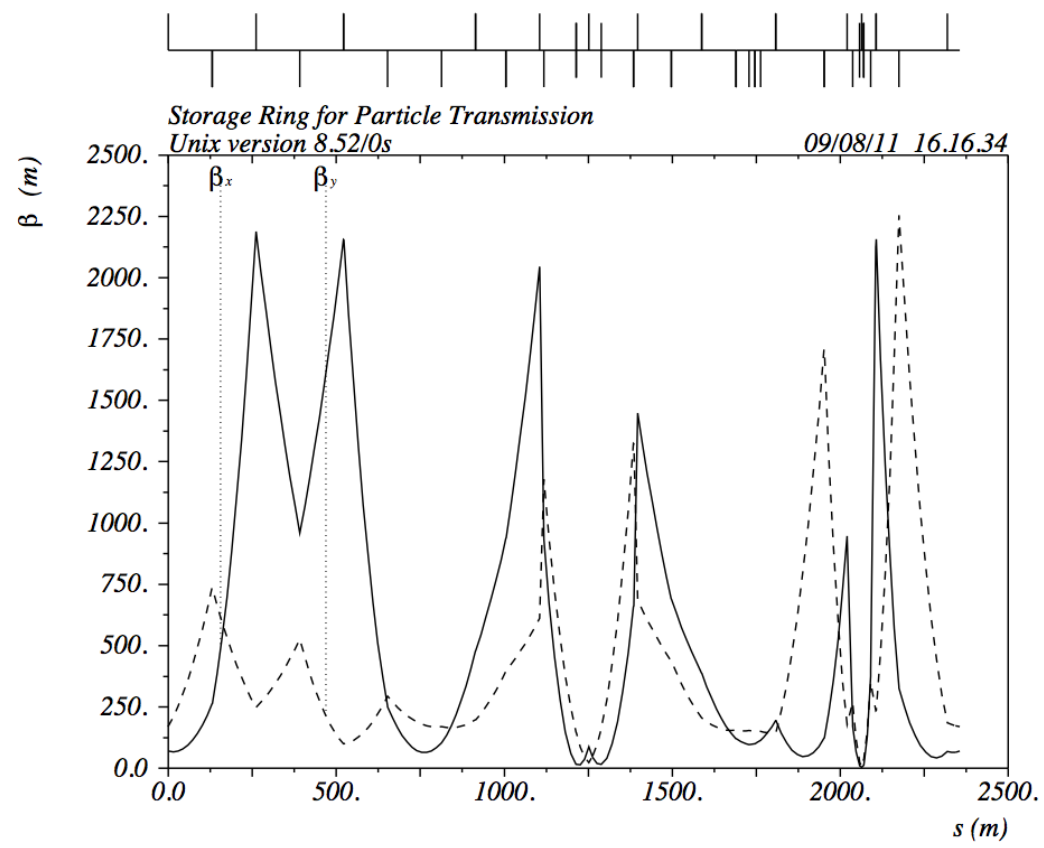

Figure 8: $\beta$ function for combined cell. 


\section{TABLES}

\begin{tabular}{|lc|lc|}
\hline Parameter & Value & Parameter & Value \\
\hline Circumference & $10,000 \mathrm{~km}$ & Dispersion $(\max )$ & $0.1 \mathrm{~m}$ \\
Beam Energy & $10 \mathrm{GeV}$ & Dipole Radius $(\min )$ & $100 \mathrm{~m}$ \\
Beam Current & $1 \mathrm{~A}$ & Dipole Field & $0.1 \mathrm{~T}$ \\
Emittance $(\varepsilon)$ & $5 \times 10^{-10} \mathrm{~m}$ & Quadrupole Gradient $(\max )$ & $10 \mathrm{~T} / \mathrm{m}$ \\
$\beta_{x, y} \max$ & $2000 \mathrm{~m}$ & $\sigma$ & $\sim 1 \mathrm{~mm}$ \\
\hline
\end{tabular}

Table 1: Parameters for the storage ring.

\begin{tabular}{ccccc}
\hline Cell & $\beta_{x}$ max. $(\mathrm{m})$ & $\beta_{y}$ max. $(\mathrm{m})$ & Dispersion max. $(\mathrm{m})$ & Total Length $(\mathrm{m})$ \\
\hline 1 & 1884.85 & 1960.75 & 0.099424 & 584.24 \\
2 & 1551.37 & 1271.54 & 0.099595 & 936.50 \\
3 & 1954.72 & 1954.72 & 0.00 & 2914.0 \\
\hline
\end{tabular}

Table 2: Maximum $\beta$ and dispersion values and the total length for the Cells in the magnet lattice. 